\title{
Obesidade: Paradigma da Disfunção Endotelial em Idade Pediátrica
}

\author{
Obesity: Paradigm of Endothelial Dysfunction in Paediatric Age Groups
}

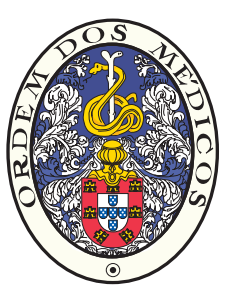

\author{
António PIRES ${ }^{1}$, Eduardo CASTELA ${ }^{1}$, Cristina SENA ${ }^{2}$, Raquel SEIÇA ${ }^{2}$ \\ Acta Med Port 2015 Mar-Apr;28(2):233-239
}

\section{RESUMO}

Introdução: A obesidade é considerada pela Organização Mundial de Saúde como uma epidemia global sendo considerada um factor de risco cardiovascular independente.

Material e Métodos: Estima-se que cerca de $10 \%$ da população pediátrica mundial apresente excesso de peso ou obesidade e que cerca de $40 \%$ sejam obesas na vida adulta. A obesidade caracteriza-se por um processo pró-inflamatório crónico que resulta na lesão do endotélio. O resultante desequilíbrio na produção de mediadores que normalmente regulam a homeostasia vascular, particularmente a biodisponibilidade do óxido nítrico, favorece um ambiente pró-aterosclerótico propício ao desenvolvimento da doença cardiovascular. Resultados: Estudos anátomo-patológicos em crianças com excesso de peso ou obesidade evidenciam lesões do endotélio que se traduzem nos precursores da lesão aterosclerótica.

Discussão: A disfunção endotelial é a manifestação mais precoce da lesão aterosclerótica. Estas alterações evidenciam-se precocemente em crianças obesas, contribuindo para a doença cardiovascular no adulto.

Conclusão: As repercussões clínicas destas alterações raramente se manifestam em idade pediátrica, sendo detectadas apenas através de biomarcadores, alterações morfológicas dos vasos ou modificações do tónus arterial periférico.

Palavras-chave: Criança; Endotélio Vascular; Obesidade Pediátrica; Tecido Adiposo.

\section{ABSTRACT}

Introduction: Obesity is considered a global epidemy with important public health issues as it is an independent risk factor in the development of cardiovascular disorders.

Material and Methods: Approximately $10 \%$ of the world's paediatric population has excess weight or obesity and $40 \%$ of these will be obese adults. Obesity is characterized by a chronic, low grade, pro-inflammatory process that ultimately results in endothelial dysfunction, the trigger lesion leading to adult cardiovascular disease. This leads to an imbalance in the synthesis of mediators that normally regulate vascular homeostasis, particularly nitric oxide bioavailability, favoring a pro-atherosclerotic status, the hallmark of cardiovascular disorders.

Results: These changes begin early in childhood and anatomopathological studies in children with excess weight or obesity have shown endothelial changes that represent the precursors of the atherosclerotic lesion.

Discussion: Endothelial dysfunction is the earliest manifestation of the atherosclerotic lesion. It is evident in obese children and, as such, it potentially contributes towards cardiovascular disease in the adult.

Conclusion: Although the clinical impact of these changes rarely manifest themselves in infancy, the presence of related biomarkers as well as vascular morphological changes can, at this early stage, be found and assessed.

Keywords: Adipose Tissue; Child; Endothelium, Vascular; Pediatric Obesity.

\section{INTRODUÇÃO}

Segundo a Organização Mundial de Saúde (OMS), em idade pediátrica, a obesidade define-se como um índice de massa corporal (IMC) superior ao percentil 95 (P95) para o sexo e a idade. ${ }^{1}$ No entanto, não é o excesso de peso, mas sim o excesso de gordura e a sua distribuição corporal que se relacionam com as comorbilidades associadas à obesidade. $^{2}$

A obesidade é considerada um fator de risco cardiovascular independente. Estima-se que o risco relativo de morte cardiovascular por cada $1 \mathrm{Kg} / \mathrm{m}^{2}$ (> IMC de 21) seja $1,10 \mathrm{e}$ 1,08 para homens e mulheres adultos, respectivamente. ${ }^{3} \mathrm{~A}$ obesidade em adolescentes é um forte preditor de doença coronária em adultos, independente de serem adultos obesos. Implicitamente, a obesidade infantil, se mantida, poderá condicionar alterações vasculares permanentes com repercussões nefastas na vida adulta.
Genericamente a obesidade classifica-se em dois subtipos: obesidade andróide (abdominal, visceral ou central) e ginóide (glúteo-femoral), sendo a obesidade central, aquela que é considerada um importante fator de risco na progressão para a doença cardiovascular (DCV). ${ }^{4}$ Daí a necessidade de introduzirmos outras medidas que espelhem mais adequadamente a adiposidade e a sua distribuição, tal como a circunferência abdominal (CA). Esta, sendo independente da estatura, reflete a quantidade de massa gorda intra-abdominal. Apesar das limitações do IMC, na população obesa existe uma correlação entre este e a percentagem de gordura corporal e o risco de DCV. ${ }^{5}$ Como tal, o IMC é considerado uma ferramenta útil e prática, permite estabelecer padrões e continua a ser a medida de referência não só no seguimento das crianças obesas mas também em estudos epidemiológicos.

1. Serviço de Cardiologia Pediátrica. Hospital Pediátrico de Coimbra. Centro Hospitalar Universitário de Coimbra. Coimbra. Portugal.

2. Laboratório de Fisiologia. Instituto de Imagem Biomédica e Ciências da Vida (IBILI). Faculdade de Medicina da Universidade de Coimbra. Coimbra. Portugal.

Recebido: 24 de Janeiro de 2014 - Aceite: 21 de Julho de 2014 | Copyright @ Ordem dos Médicos 2015 


\section{Prevalência}

A obesidade é considerada pela OMS como a epidemia do terceiro milénio, não só pela sua crescente prevalência mas pelo facto de se tratar de uma doença universal, não limitada apenas aos países desenvolvidos. Pela primeira vez, na história da humanidade, o número de pessoas com excesso de peso ultrapassou o de pessoas com desnutrição. ${ }^{6}$ Estima-se que cerca de $10 \%$ da população pediátrica mundial apresente excesso de peso ou obesidade e que cerca de $40 \%$ sejam obesas na vida adulta. ${ }^{7}$ Segundo dados fornecidos pela Organization for Economic Cooperation and Development (OECD), os valores relativos ao excesso de peso (incluindo obesidade) em Portugal, na faixa etária entre os 5 e 17 anos, encontram-se acima da média em relação a praticamente todos os países da União Europeia, especificamente $23,5 \%$ nos rapazes e $21,6 \%$ nas raparigas. $^{8}$

\section{Evolução para a doença cardiovascular: disfunção adi- pocitária e endotelial Disfunção adipocitária}

O tecido adiposo branco (TA) é um órgão endócrino, que produz inúmeras substâncias bioativas, coletivamente designadas de adipocinas. O TA tem ações metabólicas, endócrinas e imunológicas importantes e as suas células, particularmente os adipócitos, expressam e segregam diversas proteínas da fase aguda e mediadores da inflamação, tais como, o fator de necrose tumoral (TNF- $\alpha$ ), as interleucinas (IL-6, IL-8 e IL-10), o inibidor do activador do plasminogéneo (PAI-1), o angiotensinogéneo, a leptina, a adiponectina, a resistina, a lipoproteína lipase e os ácidos gordos livres. De igual forma, influenciam múltiplos processos fisiológicos contribuindo para o balanço energético, o metabolismo lipídico e glicídico, a ação da insulina, a angiogénese e a remodelação vascular, a pressão arterial e a coagulação. ${ }^{9}$

Os restantes elementos celulares constituem a fração vascular e do estroma, composta por fibroblastos, pré-adipócitos, respetivo suporte vascular e macrófagos. Os macrófagos representam cerca de $10 \%$ da celularidade do TA mas podem atingir $60 \%$ da massa celular na obesidade. Têm origem na medula óssea e o seu número está diretamente relacionado com a adiposidade. ${ }^{10}$

O aumento da adiposidade, sobretudo a visceral, e particularmente por hipertrofia dos adipócitos, tem como consequência a desregulação metabólica destas células que resulta na infiltração de macrófagos e expressão de variados mediadores inflamatórios, que se potenciam mutuamente, e que contribuem para o processo inflamatório crónico de baixo grau que caracteriza a obesidade. ${ }^{11}$

Um dos mecanismos propostos como ponto de partida para a cascata inflamatória, é o de hipoxia celular. O aumento do TA resulta na diminuição do fluxo sanguíneo à massa adipocitária contribuindo para a hipoxia e necrose celular, sobretudo dos adipócitos hipertrofiados. ${ }^{12} \mathrm{Nes}$ te contexto, é de realçar a importância da monocyte chemoattractant protein-1 (MCP-1), sobre-expressa pelo TA na obesidade, e que favorece a migração de monócitos e macrófagos para o tecido adiposo..$^{13} \mathrm{O}$ recrutamento destas células imunes tem como objetivo a fagocitose dos adipócitos necrosados. Outro mecanismo proposto relaciona-se com o excesso de ácidos gordos armazenados que resulta em anomalias funcionais do retículo endoplasmático (RE) e das mitocôndrias, culminando em stress oxidativo celular.

Os macrófagos são células metabolicamente ativas que, dependente do seu fenótipo, têm a capacidade de sintetizar substâncias pró e anti-inflamatórias, representados pelos fenótipos M1 e M2, respetivamente. Na obesidade predominam os macrófagos tipo -M1 que expressam mediadores pró-inflamatórios como, por exemplo, o TNF- $\alpha$ e a IL-6, ${ }^{14}$ que mantêm a cascata inflamatória e, como tal, são centrais no processo fisiopatológico da obesidade.

As adipocinas de maior relevo na obesidade são a leptina e a adiponectina. A leptina é uma hormona expressa predominantemente, mas não exclusivamente, pelos adipócitos. Os seus níveis circulantes relacionam-se com a quantidade de massa gorda e o tamanho do adipócito. ${ }^{15}$ Transmite ao sistema nervoso central, particularmente ao hipotálamo, informação respeitante ao teor de energia armazenada no tecido adiposo, controlando a ingestão alimentar e implicitamente o balanço energético. Paradoxalmente, na obesidade, o elevado teor de leptina não induz diminuição do apetite, traduzindo um estado de leptino-resistência. ${ }^{16}$ Para além do seu papel na homeostasia energética, a leptina está também implicada na regulação de vários mecanismos fisiológicos, entre eles, a resposta imune. ${ }^{17}$ A leptina é considerada uma adipocina com propriedades pró-inflamatórias, estimulando a secreção de outras citocinas, como é o caso da IL-6 e do TNF- $\alpha$, através da estimulação direta de células imunes como, por exemplo, os macrófagos e as células T pró-inflamatórias. Do ponto de vista clínico, em doentes com doença cardíaca confirmada, níveis elevados de leptina são preditores de futuros eventos cardiovasculares, de forma independente de outros fatores de risco cardiovascular.

A adiponectina é a mais abundante das adipocinas. Ao contrário da leptina, os seus níveis estão reduzidos na obesidade, particularmente na obesidade visceral. Atenua a expressão de TNF- $\alpha$, diminui a quimiotaxia dos macrófagos, favorece a expressão de macrófagos fenotipicamente tipo-M2, aumenta a produção de óxido nítrico, estimula a angiogénese e aumenta a sensibilidade à insulina. Daí ser uma adipocina com propriedades anti-inflamatórias, anti-aterogénicas e anti-diabéticas. A expressão desta hormona é inibida em condições de hipoxia tecidular e stress oxidativo, assim como por ação de TNF- $\alpha$ e IL-6, achados frequentes em indivíduos obesos. Como tal, o efeito anti-inflamatório da adiponectina na obesidade é suprimido. ${ }^{18}$

A ação cardioprotetora advém das suas propriedades anti-aterogénicas e anti-hipertensoras. Inibe, por um lado, a migração de células imunes para a região sub-endotelial e a ativação de várias substâncias pro-inflamatórias (moléculas de adesão vascular e intercelular) que favorecem a disfunção do endotélio e, implicitamente, estão na génese 
da formação, manutenção e rutura da placa aterogénica e, por outro, contribui para a ativação de moléculas anti-inflamatórias. ${ }^{19}$

Ou seja, quer os adipócitos quer os macrófagos do TA expressam substâncias cuja sinalização parácrina potencia localmente a libertação de múltiplos mediadores pró-inflamatórios. Estes, por si, refletem a sua atividade noutros tecidos, estimulando a libertação de outros mediadores inflamatórios, como é o caso da proteína $C$ reativa $(P C R)$ sintetizada no fígado. A desregulação na produção de citocinas pró-inflamatórias caracteriza, assim, o processo inflamatório crónico de baixo grau observado na obesidade. ${ }^{20}$ Mais ainda, estes mediadores sistémicos estão implicados nas complicações metabólicas e cardiovasculares da obesidade, incluindo a resistência à insulina, a hipertensão arterial e estados pró-trombóticos, estes dois últimos particularmente associados com o aumento do angiotensinogénio e do PAI-1, respetivamente.

\section{Disfunção endotelial}

O endotélio é uma camada unicelular que regula a homeostasia vascular através da expressão de mediadores bioativos. ${ }^{21}$ Por vias de sinalização autócrina, parácrina ou endócrina, ${ }^{22}$ estes mediadores controlam o tónus vascular (ex. óxido nítrico; endotelina-I), a agregação plaquetária (ex. Factor von Willebrand), a formação de trombos (ex. trombomodulina) e a aderência de leucócitos e facilitam a fibrinólise (ex. ativador do plasminogénio tecidular). Em condições fisiológicas, existe um equilíbrio na produção destas substâncias, estando o endotélio, no seu estado quiescente, tendencialmente vasodilatador e com características anti-aterogénicas. ${ }^{23}$ Central à normal função do endotélio, encontra-se o óxido nítrico (NO). ${ }^{24}$ Uma das principais funções do NO é promover o relaxamento do músculo liso, induzindo a vasodilatação e regulando a perfusão tecidular. Também exerce outras funções centrais à homeostasia vascular, através da inibição da adesão plaquetária e leucocitária e da proliferação de células do músculo liso vascular.

O NO é de tal forma crucial na manutenção da homeostasia vascular que a disfunção endotelial define-se em função da biodisponibilidade do NO, diminuída neste contexto, sobrepondo-se a expressão de mediadores vasoconstritores e pró-ateroscleróticos como é o caso da endotelina-1, um potente vasoconstritor de origem endotelial patente na disfunção endotelial.

Considera-se a disfunção endotelial (DE) ou ativação endotelial (termo defendido por alguns autores) a lesão mais precoce do processo aterosclerótico que precede qualquer alteração morfológica dos vasos. As alterações subsequentes levam à modificação do fenótipo da parede do vaso culminando na formação da placa aterosclerótica. Representa uma mudança do fenótipo quiescente para um mediado por citocinas inflamatórias, que contribuem para o início e a progressão da doença aterosclerótica.

Vários estudos, incluindo análises anátomo-patológicas, têm demonstrado que a doença aterosclerótica inicia- -se na infância, particularmente em crianças com fatores de risco cardiovascular. Daí que a obesidade infantil seja considerada um importante preditor de morbilidade e mortalidade cardiovascular no adulto. ${ }^{25}$

À semelhança da PCR, outras substâncias pró-inflamatórias sintetizadas pelo tecido adiposo como a resistina, a leptina, o TNF- $\alpha$ e os ácidos gordos livres, participam na lesão endotelial, diretamente actuando no endotélio e indiretamente interferindo na produção de outras adipocinas. ${ }^{26}$

Estes mediadores contribuem para a lesão endotelial através do aumento da expressão de enzimas oxidativas (ex. NADPH oxidase) formando espécies reativas de oxigénio (ROS) como o anião superóxido e o peróxido de hidrogénio. Em concentrações elevadas, as ROS são tóxicas para o ambiente celular condicionando stress oxidativo celular e consequentemente a desregulação metabólica característica da disfunção endotelial. As ROS têm a capacidade de regular a expressão de vários genes promotores de substâncias ligadas à disfunção do endotélio, como é o caso das moléculas de adesão, citocinas e fatores quimiotáticos. ${ }^{27}$ Os próprios mecanismos que contribuem para a eliminação das ROS estão comprometidos, como é o caso das enzimas antioxidantes superóxido dismutases, contribuindo para a manutenção da sinalização das reações de redução-oxidação.

No endotélio, o stress oxidativo culmina na diminuição da biodisponibilidade do NO e, consequentemente, na redução da capacidade vasodilatadora dependente do endotélio e da atividade anti-aterogénica. A produção de NO também pode estar comprometida por inibidores endógenos como é o caso da enzima dimetilarginina assimétrica (ADMA); os níveis deste antagonista competitivo da sintetase do óxido nítrico (eNOS) aumentam na presença de concentrações elevadas de TNF- $\alpha{ }^{28}$ Ou seja, a ADMA, na prática clínica, pode ser utilizada como índice indireto da biodisponibilidade do NO, visto o doseamento direto deste não ser prático dado a sua curta semi-vida.

Ainda relativamente ao stress oxidativo, e também à inflamação, é importante realçar a importância dos prostanoídes (prostaglandinas, prostaciclinas e tromboxano) e a sua relação com doenças cardiovasculares, como a hipertensão arterial e a aterosclerose. ${ }^{29}$ Estas substâncias são sintetizadas por ação enzimática das cicloxigenases (COXs), a COX-1 e a COX-2. A COX-1 existe praticamente em todos os tecidos, ao contrário da COX-2 que é sobre expressa essencialmente por estímulos pró-inflamatórios. A COX-1 é responsável pela expressão de tromboxano $A_{2}$ $\left(\mathrm{TXA}_{2}\right)$, um potente vasoconstritor que promove a adesão de plaquetas ao endotélio, e, a COX-2, pela produção da prostaciclina $\left(\mathrm{PGI}_{2}\right)$, um agente vasodilatador com ações anti-plaquetárias. $\mathrm{O}$ equilíbrio na produção destas substâncias promove a homeostasia cardiovascular, que é perturbado em processos inflamatórios, tal como a obesidade, favorecendo a expressão de TXA $_{2}$, promovendo a lesão endotelial. ${ }^{30}$

O ponto de partida para a aterosclerose inicia-se com o recrutamento de monócitos e células-T para a parede do 
vaso. ${ }^{31}$ A adesão é iniciada com o rolamento dos leucócitos no endotélio, subsequente ligação à superfície endotelial e posterior transmigração destas células inflamatórias para a camada íntima da artéria. Este processo é possível face à presença de moléculas de adesão leucocitárias expressas pelo endotélio. ${ }^{32}$ Habitualmente existem em pequenas quantidades, no entanto, na presença de citocinas inflamatórias, a síntese destas substâncias aumenta. As mais importantes são as selectinas $\mathrm{P}, \mathrm{E}$ e L, de origem plaquetária, endotelial e leucocitária, respetivamente, as moléculas de adesão intercelular (ICAM) e as moléculas de adesão das células vasculares (VCAM-1). Destas, a E-selectina relaciona-se mais especificamente com o endotélio, sendo particularmente importante no rolamento dos leucócitos. Os níveis aumentados de E-selectina associam-se a fatores de risco cardiovascular sendo um marcador precoce de ativação endotelial. ${ }^{33}$

$\mathrm{Na}$ íntima, os linfócitos e macrófagos perpetuam a resposta inflamatória. Os macrófagos englobam partículas LDL-oxidadas formando células espumosas. As moléculas de LDL- oxidadas estimulam a produção endotelial de substâncias pró-inflamatórias, incluindo moléculas de adesão leucocitária e o fator estimulador de colónias de macrófagos (M-CSF), e inibem a produção de NO. Se o processo inflamatório persistir, o recrutamento de células inflamatórias para a íntima mantem-se, aumentando assim o número de células espumosas e a expressão de citocinas que culminam na formação, manutenção e rutura da placa aterosclerótica. Também, se a inflamação se mantiver ativa, o próprio endotélio fica lesado com perda de células endoteliais para a circulação. A regeneração celular ocorre quer por proliferação de células endoteliais adjacentes, cujo potencial regenerativo é limitado, quer por migração para a circulação de células progenitoras, que têm a sua origem na medula óssea (onde representam cerca de 1-5\% da população celular) e a capacidade de se diferenciarem em células progenitoras endoteliais (EPCs). As EPCs têm um papel importante na neovascularização de orgãos isquémicos e reendotelização de vasos danificados, mantendo a integridade vascular. Nos portadores de fatores de risco cardiovascular (RCV), o número e função das EPCs encontra-se reduzido, existindo uma correlação inversa entre o número daqueles fatores presentes no mesmo individuo e o número de EPCs. ${ }^{34} \mathrm{O}$ endotélio expressa fatores de ativação, entre eles o NO, que estimulam o recrutamento de EPCs da medula óssea para a circulação periférica ocorrendo a ligação das EPCs ao endotélio, via moléculas de adesão (ex. E-selectina). ${ }^{35} \mathrm{Em}$ circunstâncias em que a biodisponibilidade do NO esteja comprometida, é de esperar um número reduzido de EPCs em circulação assim como da função destes elementos celulares. ${ }^{36}$ Estudos mostram que em eventos agudos transitórios (ex. enfarte do miocárdio) o número de EPCs encontra-se elevado. Já em patologias crónicas, os marcadores inflamatórios expressos, tal como a PCR e o TNF- $\alpha$, inibem a mobilização, diferenciação e função das EPCs. ${ }^{37}$

\section{Avaliação clínica da função endotelial}

Os conhecimentos atuais da biologia do endotélio vascular permitem a sua avaliação, tendo como base marcadores estruturais e funcionais. ${ }^{38}$

\section{Avaliação morfológica}

A avaliação estrutural e funcional do endotélio é possível através da realização da angiografia coronária, sendo este leito arterial um dos mais implicados no processo aterosclerótico. Esta técnica permite a avaliação in situ das alterações morfológicas dos vasos em questão e a resposta a fármacos vasoativos (ex. acetilcolina), permitindo aferir a biodisponibilidade do NO. No entanto, apesar de ser o teste gold standard na avaliação morfológica da DE do leito coronário, trata-se de um exame de carácter invasivo, dispendioso e associado a vários riscos que só deve ser usado mediante necessidade clínica. ${ }^{39}$ Como tal, a sua aplicabilidade como marcador da DE é limitada, particularmente em idades pediátricas.

Outro exame de interesse na prática clínica utilizada para avaliar alterações morfológicas associadas à DE, é a espessura da camada intima- média carotídea (IMT) avaliada por ultrassonografia. É um método não-invasivo, seguro e de baixo custo que permite a avaliação do risco cardiovascular, em fases precoces e avançadas da doença aterosclerótica, em grupos considerados de risco. ${ }^{40}$ Apesar de ser uma avaliação periférica, é considerada representativa do status da circulação coronária,${ }^{41}$ sendo uma importante ferramenta na avaliação da doença cardiovascular subclínica, possibilitando estratégias de prevenção. Em idades pediátricas, nos grupos de risco, o aumento do IMT também se correlaciona com a DE e, na população adulta, confere valor preditivo relativamente a futuros eventos cardiovasculares adversos e permite avaliar a evolução da doença e a resposta às terapêuticas instituídas.

O IMT pode ser avaliado em vários segmentos da artéria carótida, incluindo a carótida comum, a bifurcação carotídea, o bulbo carotídeo e a carótida interna. Apesar de alguns estudos demonstrarem que o IMT derivado da avaliação dos vários segmentos da artéria carótida apresente uma capacidade preditiva semelhante relativamente a eventos cardíacos adversos, outros autores consideram que os valores do IMT obtidos no bulbo carotídeo e na carótida interna tenham uma melhor correlação com a doença coronariana. ${ }^{42}$ Apesar do IMT ser influenciado por vários fatores de risco cardiovascular e, em particular, a hipertensão arterial, é considerado um fator independente de risco cardiovascular. ${ }^{43} \mathrm{~A}$ fim de padronizar a forma de avaliar o IMT, o Mannheim Carotid Intima-Media Thickness Consensus sugeriu que este fosse avaliado na parede distal da artéria carótida comum ${ }^{44}$. Outra limitação desta técnica refere-se à inexistência de valores de referência ou pontos de corte ajustados à idade, género e etnia, que atribuam risco cardiovascular.

\section{Avaliação funcional}

O facto de a DE ser uma doença sistémica possibilita- 
-nos a avaliação não-invasiva da vasorreatividade fluxo-dependente (NO dependente) em artérias mais periféricas, como é o caso da artéria braquial. ${ }^{45}$ Neste contexto, utiliza-se a ultrassonografia braquial para avaliar a alteração do diâmetro do vaso, antes e após oclusão do fluxo braquial pela insuflação de uma braçadeira, ou seja, através da hiperémia reativa ou vasodilatação-fluxo-mediada (FMV). ${ }^{46}$ Considera-se uma técnica sensível pois relaciona-se com a função endotelial das coronárias, mas com limitações de ordem técnica e interpretativa, pelo que, segundo alguns autores, não é um método útil para estratificação de risco. ${ }^{47}$ Nas crianças, a sua utilização é limitada pelo potencial desconforto imposto (insuflação da braçadeira $50 \mathrm{mmHg}$ acima da tensão arterial sistólica durante cinco minutos) e necessidade de colaboração. ${ }^{48}$

Outro método não-invasivo de utilidade na prática clínica, principalmente em idades pediátricas, face à sua fácil aplicabilidade e por existirem valores de referência para idades pediátricas, ${ }^{49,50}$ é a avaliação da velocidade da onda de pulso (VOP), que permite a avaliação indireta da rigidez arterial. A VOP quantifica o tempo que a onda de pulso leva a percorrer uma determinada distância no leito arterial. Estudos mostram que existe uma correlação entre a VOP e a presença de DE. ${ }^{51}$ Quanto maior a rigidez do vaso, e implicitamente uma menor biodisponibilidade do NO, mais rápida a VOP. ${ }^{52,53}$ Para além de potencialmente identificar doença subclínica, também tem valor preditivo relativamente a futuros eventos cardiovasculares adversos.

\section{Avaliação analítica}

A quantificação de biomarcadores relacionados com a DE permite avaliar alguns aspetos fundamentais do processo fisiopatológico subjacente, nomeadamente a cascata inflamatória, a ativação do endotélio, a biodisponibilidade do $\mathrm{NO}$ e os mecanismos reparadores do endotélio.

\section{Marcadores inflamatórios}

$\mathrm{Na}$ obesidade, vários marcadores inflamatórios são expressos, sendo a proteína C-reativa ultra-sensível (PCRus) a de maior utilidade na rotina clínica. ${ }^{54} \mathrm{~A}$ PCR tem uma semivida de cerca 18 horas, não sendo influenciada pela idade, o estádio de Tanner ou o ciclo circadiano. É um biomarcador sensível, mas com baixa especificidade para inflamação tecidual. Vários estudos têm demonstrado a sua relação com as doenças cardiovasculares sendo considerado um preditor independente de eventos cardiovasculares. ${ }^{55,56} \mathrm{Na}$ prática clínica, o seu interesse prende-se com o facto de se poder atribuir risco relativo de doença aterosclerótica, mesmo em indivíduos aparentemente saudáveis, nomeadamente baixo (<0,1 mg/dl), moderado $(0,1-0,3 \mathrm{mg} /$ dl) e elevado (> 0,3 mg/dl). Portadores de PCRus superior a $0,3 \mathrm{mg} / \mathrm{dl}$ têm duas vezes mais a probabilidade de virem a ter doença aterosclerótica, comparado com indivíduos com baixo risco (Classe de recomendação lla e nível de evidência B). ${ }^{57,58} \mathrm{Em}$ crianças obesas, a PCRus não só traduz a presença de um processo inflamatório persistente mas, à semelhança da população adulta, também confere risco cardiovascular. ${ }^{59,60}$

\section{Marcadores da ativação endotelial}

As moléculas de adesão celular facilitam a ligação de leucócitos ao endotélio e, posteriormente, a sua transmigração para o espaço sub-endotelial. As mais estudadas são as da família das selectinas e as da superfamília das imunoglobulinas, a VCMA e a ICAM. Conforme referido, a E-selectina tem características singulares, visto derivar de células endoteliais, ao contrário das restantes, que têm múltiplas fontes. É expressa precocemente em resposta a um estímulo inflamatório e, como tal, é considerada um indicador precoce da ativação endotelial, podendo, potencialmente, aferir RCV. ${ }^{61,62} \mathrm{Em}$ quadros inflamatórios mantidos, como é o caso da obesidade, é de esperar a sua sobre expressão. ${ }^{63}$

\section{Marcadores da biodisponibilidade do NO}

A avaliação direta do NO plasmático, no contexto da $\mathrm{DE}$, é condicionada por vários fatores, especificamente a semivida extremamente curta do NO e pelo facto do NO não ser expresso exclusivamente pelo endotélio. ${ }^{64}$ Logo, a necessidade de se recorrer a uma avaliação indireta, através do doseamento de metabolitos do NO (que pelos mesmos motivos podem não ser representativos do NO endotelial) ou do já referido inibidor endógeno competitivo da eNOS, a ADMA. ${ }^{65}$ Os níveis desta enzima, para além de estarem elevados em portadores de doenças com risco cardiovascular ${ }^{66}$ incluindo a obesidade,${ }^{67}$ são preditivos de eventos cardiovasculares adversos ${ }^{68}$ que se correlacionam com outros marcadores de RCV, como é o caso da IMT ${ }^{69} \mathrm{e}$ da PCR. ${ }^{70}$

\section{Marcadores dos mecanismos reparadores}

Em 1997, Asahara et al descreveram, pela primeira vez, a presença de células mononucleares CD34 positivas (de origem estaminal) na circulação periférica com capacidade de neovascularização, às quais atribuíram o nome de células progenitoras endoteliais (EPCs). ${ }^{71}$ Atualmente sabe-se que as EPCs, de facto, englobam várias populações celulares, sendo a denominação de EPCs atribuída às mais importantes.

As EPCs têm a capacidade de se diferenciar em células endoteliais maduras e têm um importante papel na reparação do endotélio lesado. ${ }^{72} \mathrm{~A}$ libertação destas células da medula óssea para a circulação periférica ainda não está completamente esclarecida, propondo-se, entre outros mecanismos, a ativação da metaloproteinase da matriz -9 (MMP-9), um processo NO dependente. As EPCs expressam alguns recetores comuns à célula endotelial, nomeadamente o Cluster of Differentiation 34 (CD34) e o Vascular Endothelium Growth Factor Receptor-2 (VEGFR2-), que permitem a sua ligação ao endotélio danificado e a sua quantificação por citometria de fluxo. ${ }^{73,74}$ Estes marcadores não são exclusivos da célula endotelial, podendo condicionar potenciais erros na sua leitura. No entanto, apesar de não existir um marcador específico, a associação de 
CD34-CD33-VEGFR2 na mesma linha celular aparenta caracterizar as EPCs. ${ }^{75}$

\section{CONCLUSÃO}

Em crianças portadoras de factores de risco cardiovascular, particularmente a obesidade pela sua prevalência, já são patentes as alterações mais precoces da doença aterosclerótica. Nestas faixas etárias estas alterações são reversíveis, daí ser, de todo o interesse a implementação de medidas que evitem a progressão da doença. Na prática clínica o acesso a exames complementares de diagnóstico,

\section{REFERÊNCIAS}

1. Slyper $\mathrm{AH}$. The pediatric obesity epidemic: causes and controversies. J Clin Endocrinol Metab. 2004;89:2540-7

2. Sweeting $\mathrm{H}$. Measurement and definitions of obesity in childhood and adolescence: a field guide for the uninitiated. Nutr J. 2007;6:32.

3. Stevens J, Pamuk ER, Williamson DF, Thun MJ, Wood JL. The effect of age on the association between body-mass index and mortality. $\mathrm{N}$ Engl J Med. 1998;338:1-7.

4. Monteiro S. Obesidade: um olhar sobre a sua fisiopatologia e os novos marcadores de risco cardiovascular. Rev Port Cardiol. 2010;29:S39-47.

5. Gray D, Fujioka K. Use of relative weight and body mass index for the determination of adiposity. J Clin Epidemiol. 1991:44;545-50.

6. Gomes F, Telo DF, Souza HP, Souza HP, Nicolau JC, Halpern A, et al. Obesidade e doença arterial coronariana: papel da inflamação vascular. Arq Bras Cardiol. 2010;94:273-9.

7. Bessesen DH. Update on obesity. J Clin Endocrinol Metab. 2008:93;2027-34.

8. OECD. Overweight and obesity among children, in Health at a Glance 2011: OECD indicators, OECD publishing; [consultado em 2014 April 15]. Disponível em: http://dx.doi.org/10.1787/health_glance-2011-19en.

9. Kersshaw EE, Flier JS. Adipose tissue as an endocrine organ. J Clin Endocrinol Metab. 2004;89:2548-56.

10. Berg AH, Scherer PE. Adipose tissue, inflammation, and cardiovascular disease. Circ Res. 2005;96:939-49.

11. Ramalho R, Guimarães C. Papel do tecido adipose e dos macrófagos no estado de inflamação crónica associada à obesidade. Implicações clínicas. Acta Med Port. 2008;21:489-96.

12. Trayhurn $P$, Wang $B$, Wood IS. Hypoxia in adipose tissue: a basis for the dysregulation of tissue function in obesity. Br J Nutr. 2008;100:227-35.

13. Rasouli N, Kern PA. Adipocytokines and the metabolic complications of obesity. J Clin Endocrinol Metab. 2008;93:s64-73.

14. Lumeng CN, Bodzin JL, Saltiel AR. Obesity induces a phenotypic switch in adipose tissue macrophage polarization. J Clin Invest. 2007;117:17584.

15. Margetic S, Gazzola C, Peggi GG, Hill RA. Leptin: a review of its peripheral actions and interactions. Int $\mathrm{J}$ Obes Relat Metab Disord. 2002;26:1407-33.

16. Meyers MG, Cowley MA, Munzberg H. Mechanisms of leptin action and leptin resistance. 2008. Annu Rev Physiol. 2008;70:537-56.

17. Sousa M, Brás-Silva $C$, Leite-Moreida A. O papel da leptina na regulação da homeostasia energética. Acta Med Port. 2009;22:291-8.

18. Aprahamian TR, Sam F. Adiponectin in cardiovascular inflammation and obesity. Int J Inflam. 2011;2011:376909.

19. Quedraogo R, Gong Y, Berzins B, Wu X, Mahadev K, Chan L, et al. Adiponectin deficiency increases leucocyte-endothelium interactions via upregulation of endotelial cell adhesion moleculesin vivo. J Clin Invest. 2007;117:1718-26.

20. Shimabukuro M, Higa N, Asahi T, Oshiro Y, Taqawa T, Ueda S, et al. Hypoadenopectinemia is closely linked to endothelial dysfunction in man. J Clin Endocrinol Metab. 2003;88:3236-40.

21. Dandona P, Aljada A, Chaudhuri A, Mohanty P. Endothelial dysfunction, inflammation and diabetes. Rev Endocr Metab Disord. 2004;5:189-97.

22. Deanfield JE, Halcox JP, Rabelink TJ. Endothelial function and dysfunction. Testing and clinical relevance. Circulation. 2007;115:128595.

23. Bahia L, de Aguiar LGK, Villela NR, Bottino D, Bouskela E. O endotélio na síndrome metabólica. Arq Bras Endocrinol Metab. 2006;50:291-303.

24. Verma S, Anderson TJ. Fundamentals of endothelial function for the clinical cardiologist. Circulation. 2002;105:546-9. para além da sua utilidade na aferição de risco, permitem avaliar a resposta às medidas terapêuticas instituídas.

\section{CONFLITOS DE INTERESSE}

Os autores declaram não ter qualquer conflito de interesse relativamente ao presente artigo.

\section{FONTES DE FINANCIAMENTO}

Os autores declaram não ter recebido qualquer subsídio relativo ao presente artigo.

25. Berenson GS, Srinivasan SR, Bao W, Newman WP, Tracy RE, Wattigney WA, et al. Association between multiple cardiovascular risk factors and atherosclerosis in children and young adults. N Eng J Med. 1998;338:1650-56.

26. Pasceri V, Willerson JT, Yeh ET. Direct proinflamatory effect of Creactive protein on human endothelial cells. Circulation. 2000;102:21658.

27. Kapiotis S, Holzer G, Schaller G, Haumer M, Widhalm H, Weqhuber $\mathrm{D}$, et al. A proinflammatory state is detectable in obese children and is accompanied by functional and morphological vascular changes. Arterioscler Thromb Vasc Biol. 2006;26:2541-6.

28. Avogaro A, de Kreutzenberg SV. Mechanisms of endothelial dysfunction in obesity. Clin Chim Acta. 2005;360:9-26.

29. Smyth E, Grosser T, Wang M, Yu Y, FitzGerald G. Prostanoids in health and disease. J Lipid Res. 2009;50:S423-8.

30. Capra V, Angiolillo DJ, Cattaneo M, Sakariassen KS. Impact of vascular thromboxane prostanoid receptor activation on hemostasis, thrombosis, oxidative stress and inflammation. J Thromb Haemost. 2014;12:126-37.

31. Giribela CR, Gengo R, Hong V, Consolim-Colombo FM. Função e disfunção endotelial: da fisiopatologia às perspectivas de uso em pesquisa e na prática clínica. Rev Bras Hipertens. 2011;18:27-32.

32. Szmitko PE, Wang $\mathrm{CH}$, Weisel RD, de Almeida JR, Anderson TJ, Verma S. New markers of inflammation and endothelial cell activation. Circulation. 2003;108:1017-23.

33. Hwang SJ, Ballantyne CM, Sharret AR, Smith LC, Davis CE, Gotto AM, et al. Circulating adhesion molecules VCAM-1, ICAM-1, and E-selectin in carotid atherosclerosis and incident coronary heart disease cases. The Atherosclerosis Risk in Communities (ARIC) study. Circulation. 1997;96:4219-25.

34. Tepper OM, Galiano RD, Capla JM, Kalka C, Gaqne PJ, Jacobowitz $\mathrm{GR}$, et al. Human endothelial progenitor cells from type II diabetes exhibit impaired proliferation, adhesion and incorporation into vascular structures. Circulation. 2002;106:2781-6.

35. Guthrie SM, Curtis LM, Mames RN, Simon GG, Grant MB, Scott EW. The nitric oxide pathway modulates hemangioblast activity of adult hemapoetic stem cells. Blood. 2005;105:1916-22.

36. Lin CP, Lin FYL, Huang $\mathrm{PH}$, Chen $\mathrm{YL}$, Chen WC, Chen HY, et al. Endothelial progenitor cell dysfunction in cardiovascular diseases: role of reactive oxygen species and inflammation. Biomed Res Int. 2013;2013:845037.

37. Tsai TH, Chai HT, Sun CK, Yen CH, Leu S, Chen YL, et al. Obesity suppresses circulating level and function of endothelial progenitor cells and heart function. J Trans Med. 2012;10:137.

38. Deanfield J, Donald A, Ferri C, Giannattasio C, Halcox J, Halligan S, et al. Working group of endothelium and endothelial factors of the European Society of Hypertension. Endothelial function and dysfunction. Part I. Methodological issues for assessment in the different vascular beds: a statement by Working group of endothelium and endothelial factors of the European Society of Hypertension. Circulation. 2005;105:3017-24.

39. Ribeiro F, Alves AJ, Teixeira M, Ribeiro V, Duarte JA, Oliveira J. Funcionalidade endotelial e aterosclerose: marcadores sanguíneos com utilidade clínica. Rev Port Cardiol. 2009;28:1121-51.

40. Schiel R, Beltschikow W, Radon S, Kramer G, Perenthaler T, Stein G. Increased carotid intima-media thickness and associations with cardiovascular risk factors in obese and overweight children and adolescents. Eur J Med Res. 2007;12;503-8.

41. Huang K, Zou CC, Yang XZ, Chen XQ, Liang L. Carotid intima-media thickness and serum endothelial marker levels in obese children with metabolic syndrome. Arch Pediatr Adolesc Med. 2010;164:846-51. 
42. Torres FS, Moreira CM, Vianna FF, Gus M. Medida da espessura das camadas íntima e média das artérias carótidas para avaliação do risco cardiovascular. Rev Bras Hipertens. 2007;14:167-71.

43. Mukherjee D, Yadav JS. Carotid artery intimal-medial thickness: indicator of atherosclerotic burden and response to risk factor modification. Am Heart J. 2002;144:753-9.

44. Touboul PJ, Hennerici MG, Meairs S, Adams H, Amarenco P, Bornstein $\mathrm{N}$, et al. Mannheim carotid intima-media thickness consensus (20042006). Cerebrovasc Dis. 2007;23:75-8.

45. Santos S, Vasconcelos D, Leite L. Função endotelial - avaliação pela reatividade braquial. Rev Bras Ecocardiogr Imagem Cardiovasc. 2010;23:50-4.

46. Corretti MC, Anderson TJ, Benjamin EJ, Celermajer D, Charbonneau $F$, Creager MA, et al. Guidelines for the ultrasound assessment of endothelial-dependent flow-mediated vasodilation of the brachial artery: a report of the International Brachial Artery Reactivity Task Force. J Am Coll Cardiol. 2002;39:257-65.

47. Celemajer DS. Reliable endothelial function testing at our fingertips? Circulation. 2008;117:2428-30.

48. Rosenthal DN, Chin C. Brachial artery reactivity:a modified technique with applicability to children. J Am Soc Echocardiogr. 1999;12:850-2.

49. Reusz GS, Cseprekal O, Temmar M, Kis E, Cherif AB, Fekete A, et al. Reference values of pulse wave velocity in healthy children and teenagers. Hypertension. 2010;56:217-24.

50. Jadhav UM, Kadam NN. Non-invasive assessment of arterial stiffness by pulse-wave velocity correlates with endothelial dysfunction. Indian Heart J. 2005;57:226-32

51. Naka K, Tweddel AC, Doshi SN. Flow-mediated changes in pulse wave velocity: a new clinical measure of endothelial function. Eur Heart J. 2006;27:302-9.

52. Reusz GS, Csepekral O, Temmar M, Kis E, Cherif AB, Thaleb A, et al. Reference values of pulse wave velocity in healthy children and teenagers. Hypertension. 2010;56:217-24.

53. Vlachopoulos C, Aznaouridis K, Stefanadis C. Prediction of cardiovascular events and all-cause mortality with arterial stiffness: a systematic review and meta-analysis. J Am Coll Cardiol. 2010:55:131827.

54. Filho AC, Araújo RG, Galvão TG, Chagas AC. Inflamação e aterosclerose: integração de novas teorias e valorização dos novos marcadores. Rev Bras Cardiol Invas. 2003;11:14-9.

55. Pasceri V, Willerson JT, Yeh ET. Direct proinflammatory effect of C-reactive protein on human endothelial cells. Circulation. 2000;102:2165-8.

56. Rifai N, Ridker PM. Proposed cardiovascular risk assessment algorithm using high-sensitivity C-Reactive Protein and lipid screening. Clin Chem. 2001; $47: 28-30$

57. Ridker PM. High-sensitivity C-Reactive Protein: potential adjunct for global risk assessment in the primary prevention of cardiovascular disease. Circulation. 2001;103:1813-8.

58. Pearson TA, Mensah GA, Alexander RW, Anderson JL, Cannon III $\mathrm{RO}$, Criqui $\mathrm{M}$, et al. Markers of inflammation and cardiovascular disease application to clinical and public health practice. A statement for healthcare professionals from the Centers for Disease Control and Prevention and the American Heart Association. Circulation. 2003;107:499-511.

59. Soriano-Guilén L, Hernández-Garcia B, Pita J, Dominguez-Garrido N,
Rio-Camacho D, Rovira A. High-sensitivity C-reactive protein is a good marker of cardiovascular risk in obese children and adolescents. Eur J Endocr. 2008;159:R1-4.

60. Hiura M, Kikuchi T, Nagasaki K, Uchiyama M. Elevation of serum C-reactive protein levels is associated with obesity in boys. Hypertens Res. 2003;26:541-6

61. Walzog B, Gaehtgens P. Adhesion molecules: the path to a better understanding of acute inflammation. Physiology. 2000;15:107-13.

62. Ferri C, Desideri G, Valenti N, Bellini C, Pasin M, Santucci A, et al. Early upregulation of endotelial adhesion molecules in obese hypertensive men. Hypertension. 1999;34:568-73.

63. Glowinskal B, Urban M, Peczynska J, Florys B. Soluble adhesion molecules (sICAM-1, sVCAM-1) and sE-selectins (sE selectin, sP selectin, sL selectin) levels in children and adolescents with obesity, hypertension, and diabetes. Metabolism. 2005;54:1020-6.

64. Tousoulis D, Kampoli AM, Tentolouris C, Papageorgiou N, Stefanadis C. The role of nitric oxide on endothelial function. Curr Vasc Pharmacol. 2012;10:4-18.

65. Boger RH, Bode-Boger SM, Szuba A, Tsao PS, Chan JR, Tangphao $\mathrm{O}$, et al. Asymmetric dimethylarginine (ADMA): a novel risk factor for endothelial dysfunction: its role in hypercholesterolemia. Circulation. 1998;98:1842-7

66. Boger $\mathrm{RH}$. The emerging role of asymetric dimethylarginine as a novel cardiovascular risk factor. Cardiovasc Res. 2003;59:824-33.

67. Gruber HJ, Mayer C, Meinitzer A, Almer G, Horejsi R, Moller R, et al. Asymetric dimethylarginine (ADMA) is tightly correlated with growth in juveniles without correlations to obesity related disorders. Exp Clin Endocrinol Diabetes. 2008;116:520-4.

68. Valkonen VP, Paiva H, Salonen JT, Lakka TA, Lehtimaki T, Laakso $\mathrm{J}$, et al. Risk of acute coronary events and serum concentration of asymmetrical dimethylarginine. Lancet. 2001;358:2127-8.

69. Furuki $\mathrm{K}$, Adachi $\mathrm{H}$, Matsuoka H, Enomoto $\mathrm{M}$, Satoh $\mathrm{A}$, Hino A, et al. Plasma levels of asymmetric dimethylarginine (ADMA) are related to intima-media thickness of the carotid artery: an epidemiological study. Atherosclerosis. 2007;191:206-10.

70. Tripepi G, Raso FM, Sijbrands E, Seck MS, Mass R, Boger R, et al. Inflammation and asymetric dimethylarginine for predicting death and cardiovascular events in ESRD patients. Clin J Am Soc Nephrol. 2011:6:1714-21.

71. Du F, Zhou J, Gong R. Endothelial progenitor cells in atherosclerosis. Front Biosci. 2012;17:2327-49.

72. Sutherland DR, Keeney AL, Navar R, Nayar R, Chin-Yee I. The ISHAGE guidelines for CD34+ cell determination by flow cytometry. International Society of Hemotherapy and Graft Engineering. J Hematother. 1996;5:213-26.

73. Hirschi KK, Ingram DA, Yoder MC. Assessing identity, phenotype, and fate of endothelial progenitor cells. Arterioscler Thromb Vasc Biol. 2008;28:1584-95.

74. Arnold C, Wenta D, Muller-Ehmsen J, Sreerman N, Graf C. Progenitor cell number is correlated to physical performance in obese children and young adolescents. Cardiol Young. 2010;20;381-6.

75. Jung $C$, Fischer $N$, Fritzenwanger $M$, Thude $H$, Ferrari $M$, Fabris $M$, et al. Endothelial progenitor cells in adolescents: impact of overweight, age,smoking, sport and cytokines in younger age. Clin Res Cardiol. 98:179-88. 
António PIRES, Eduardo CASTELA, Cristina SENA, Raquel SEIÇA

\section{Obesidade: Paradigma da Disfunção Endotelial em Idade Pediátrica}

Acta Med Port 2015:28:233-239

Publicado pela Acta Médica Portuguesa, a Revista Científica da Ordem dos Médicos

Av. Almirante Gago Coutinho, 151

1749-084 Lisboa, Portugal.

Tel: +351218428 215

E-mail: submissao@actamedicaportuguesa.com

www.actamedicaportuguesa.com

ISSN:0870-399X | e-ISSN: 1646-0758

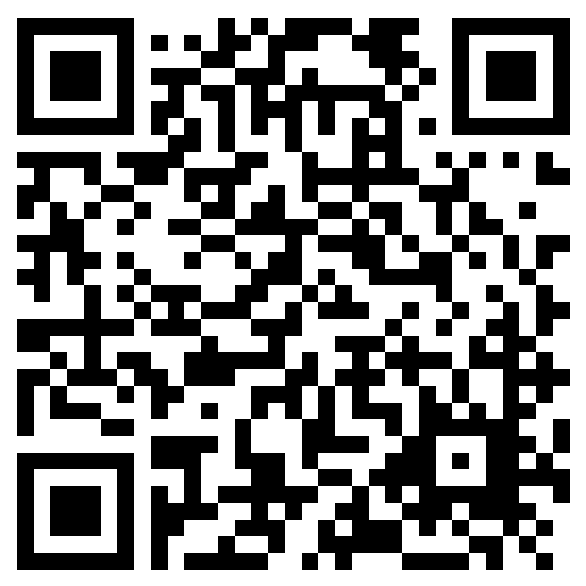

